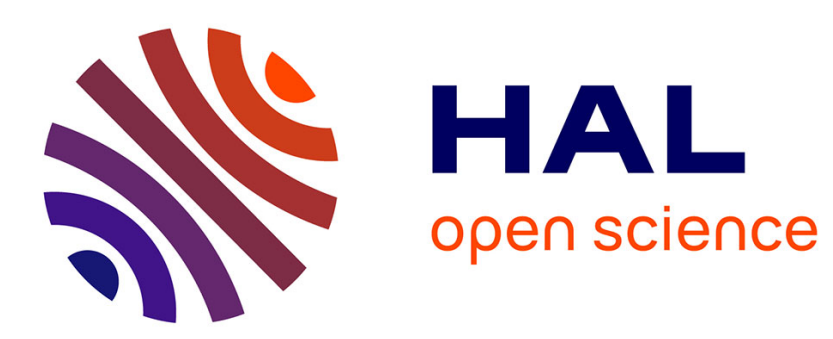

\title{
Tactile sensing for manipulation
}

Qiang Li, Lorenzo Natale, Robert Haschke, Andrea Cherubini, Anh-Van Ho, Helge Ritter

\section{To cite this version:}

Qiang Li, Lorenzo Natale, Robert Haschke, Andrea Cherubini, Anh-Van Ho, et al.. Tactile sensing for manipulation. International Journal of Humanoid Robotics, 2018, 15 (01), pp.1802001. 10.1142/S0219843618020012 . hal-01736947

\section{HAL Id: hal-01736947 https://hal.science/hal-01736947}

Submitted on 19 Mar 2018

HAL is a multi-disciplinary open access archive for the deposit and dissemination of scientific research documents, whether they are published or not. The documents may come from teaching and research institutions in France or abroad, or from public or private research centers.
L'archive ouverte pluridisciplinaire HAL, est destinée au dépôt et à la diffusion de documents scientifiques de niveau recherche, publiés ou non, émanant des établissements d'enseignement et de recherche français ou étrangers, des laboratoires publics ou privés. 


\title{
Tactile sensing for manipulation
}

\author{
Qiang Li (CITEC/Bielefeld University,Germany) \\ Lorenzo Natale (iCub Facility/Istituto Italiano di Tecnologia(IIT), Italy) \\ Robert Haschke (CITEC/Bielefeld University, Germany) \\ Andrea Cherubini (LIRMM/Universitéde Montpellier, France) \\ Anh-Van Ho (Japan Adv. Institute of Science and Technology, Japan) \\ Helge Ritter (CITEC/Bielefeld University, Germany)
}

Tactile sensing technology has achieved much progress in last decades. Recently developed tactile sensing systems have been utilized for implementation of bodily-aware robots, enhancement of robotic hand's dexterous manipulation, development of wearable devices for facilitating the human robot interaction, and so on. While tactile sensing has resulted in many possibilities in robotic research, robot's manipulation is still far from that of its counterpart-human- in term of dexterity. To overcome this issue, we are considering the following three aspects that need to be tackled.

- Available tactile sensing technology is struggling to meet the capabilities of humans' sense of touch;

- Tactile data processing algorithms and data-driven learning technology are still immature;

- General and robust robot manipulation control frameworks are missing.

In this special issue, we solicited original papers from different domains, e.g. tactile hardware development, robotic manipulation, and machine learning to ignite in-depth discussion of current progress and future challenge of tactile-driven manipulation, and reshape the potential link among such interdisciplinary research fields. After a strict peer-review process, 6 papers are accepted for publication in this special issue. The key topics are summarized as follows:

- How to design a robust grasp controller that exploits tactile information on the robot hand?

- How to design a tactile-based exploration strategy to extract an object's physical properties (shape, pose and compliance)?

- How to recognize objects via tactile-related multi-modality properties using advanced machine learning method?

E. Torres-Jara and N.Lorenzo propose a robust method to grasp and insert an object which is not held by other fixtures. The whole grasp procedure is as 
follows: (1) the robot hand uses a tactile sensor for initializing a contact to check the object's presence. (2)the robot hand forms a pre-grasp posture by embracing the object by the fingers. (3)the robot hand stably lifts the object by detecting whether slippery happens. (4)the robot hand places the object safely by confirming that the object is supported by a table. For the insertion task, an event of changed supported contact force is detected by estimating shear force from tactile sensor. The authors demonstrate the method's robustness with real robot experiment.

Y.T.Ding et. al. propose a particle filter-based method to estimate grasped object's pose exploiting tactile sensing and haptic rendering model (HRM). In this work, the main contribution is to define an innovative cost function-distance between a measured and predicted tactile image feature from a given object's model, to update the particles weight. The object pose's accuracy is evaluated by a grasping scenario-a 3D printed hand equipped with low cost tactile sensor matrix grasps a known object.

The papers from C. Rosales et. al. and S.Ottenhaus et.al. are both focusing on the use of the tactile exploration to recover the shape of an unknown object. The former employs the Gaussian Processes to represent object's surface and predicting the uncertainty of unexplored area. A planning method-AtlasRRT is used to compute the path to explore the region of greatest uncertainty. The latter highlights that the object can be reconstructed by sparse contact information and high-efficiency exploration strategy. The selection of exploration actions depends on the estimated information gain and on the expected costs of exploration actions.

T.Bhattacharjee et. al. discuss objects' classification based on the objects' tactile temporal properties extracted by a moving tactile sensor. Comparing with their previous work, the authors in this paper are focusing on analyzing the generalization of classification method. More concretely, which kind of tactile-driven method is more robust if the tested contact velocity and arm stiffness are not the same with these parameters used for sampling of training data.

M.Kaboli et. al. propose an active tactile transfer learning method for improving the efficiency of object's classification. To this end, prior knowledge about the object properties has been exploited and the most relevant one is selected. The experiments showed that the robot successfully discriminates among new objects with $72 \%$ discrimination accuracy using only one training sample.

\section{Acknowledgements}

This Special Issue was inspired by a workshop on Tactile sensing for manipulation held at IEEE Humanoids 2016, and we would also like to thank all the attendees joining and discussing in the workshop. We also thank for contributions from all authors submitted to this special issue and for efforts from reviewers and editors of the International Journal of Humanoid Robotics. 